\title{
Perspective
}

PERSPECTIVE Actualité en histoire de l'art

1 | 2009

Antiquité/Moyen Âge

\section{La sculpture grecque : découvertes archéologiques, avancées scientifiques, orientations \\ méthodologiques}

Greek sculpture: archaeological discoveries, scientific advances, methodological orientations

Irene Bald Romano, Reinhard Senff, Emmanuel Voutiras et Antoine Hermary

\section{OpenEdition}

Journals

Édition électronique

URL : http://journals.openedition.org/perspective/1438

DOI : $10.4000 /$ perspective. 1438

ISSN : 2269-7721

Éditeur

Institut national d'histoire de l'art

Édition imprimée

Date de publication : 31 mars 2009

Pagination : 10-22

ISSN : 1777-7852

Référence électronique

Irene Bald Romano, Reinhard Senff, Emmanuel Voutiras et Antoine Hermary, «La sculpture grecque découvertes archéologiques, avancées scientifiques, orientations méthodologiques », Perspective [En ligne], 1 | 2009, mis en ligne le 22 juillet 2014, consulté le 01 octobre 2020. URL : http://

journals.openedition.org/perspective/1438; DOI : https://doi.org/10.4000/perspective.1438 
Antiquité

10 DÉBAT

23 TRAVAUX

57 ACTUAlité 


\section{La sculpture grecque : découvertes archéologiques, avancées scientifiques, orientations méthodologiques}

Points de vue d'Irene Bald Romano, Reinhard Senff et Emmanuel Voutiras, avec Antoine Hermary

\author{
Antoine Hermary, professeur \\ d'archéologie et de civilisation \\ grecques à l'Université de \\ Provence, a travaillé en particu- \\ lier sur les sculptures de Délos \\ et de Chypre. \\ Directeur administratif de \\ I'American School of Classical \\ Studies à Athènes, Irene Bald \\ Romano a publié de nombreux \\ catalogues de musées sur la \\ sculpture classique \\ ainsi que des articles sur les \\ premières images de culte du \\ monde grec.
}

Directeur associé du DAI à Athènes, Reinhard Senff dirige les campagnes de fouilles sur le site d'Olympie. Il a réalisé des chantiers de fouilles à Milet et effectué des recherches sur la sculpture et l'architecture de Chypre et de Grèce orientale.

Professeur d'archéologie classique à l'Université de Thessalonique, Emmanuel Voutiras est membre de la Society for the Promotion of Hellenic Studies de Londres, de la Société Archéologique d'Athènes, du Centre International d'Étude de la Religion Grecque Ancienne (CIERGA) de Bruxelles et membre correspondant du DAI. Il travaille principalement sur le portrait, l'épigraphie et la religion de la Grèce antique.
Commençons ce débat par une remarque de vocabulaire qui en dit long sur les traditions historiographiques et les enjeux actuels de la recherche. L'emploi, dans les questions, du terme "sculpture » (en français et en anglais) a orienté les réponses vers les œuvres en marbre et en bronze, alors que l'expression " plastique grecque », plus générale mais peu usitée en français et en anglais - contrairement au mot Plastik en allemand-aurait peut-être entraîné davantage de remarques sur l'apport des découvertes et des recherches concernant les sculptures et figurines en terre cuite.

L'époque des "grandes fouilles" - en particulier celles des grands sanctuaires de Grèce continentale (I'Acropole d'Athènes, Delphes), du Péloponnèse (Olympie) et des îles égéennes (Délos, Samos) - étant passée depuis longtemps, les années récentes ne sont pas caractérisées par une abondance de découvertes. Excepté les statues archaïques mises au jour dans le cimetière du Céramique à Athènes, ce sont moins les marbres que les bronzes qui, pour les sculptures de grandes dimensions, constituent les nouveautés les plus spectaculaires : après les guerriers de Riace, qui ont permis de lancer sur de nouvelles bases les études techniques sur la statuaire grecque en bronze dans les années 1970 (rappelons, sur ce point, l'apport fondamental de Claude Rolley, récemment disparu), d'autres œuvres sont apparues, souvent au hasard de découvertes sous-marines (Le Satyre de Mazara del Vallo en Sicile, L'Apoxyomène repêché près des côtes croates). Les recherches sur les bronzes connaîtront sans aucun doute d'autres développements au cours des prochaines années. Ces découvertes, et d'autres, ne sont pas (encore) à l'origine des principaux changements d'orientation des années 1990 et 2000, caractérisés par un intérêt renouvelé pour les styles régionaux - notamment depuis les études de Francis Croissant - et pour les aspects techniques de la sculpture, en particulier l'origine des marbres - avec les colloques d'ASMOSIA (The Association for the Study of Marble and Other Stones in Antiquity) et le décor polychrome des œuvres, domaine dans lequel Vinzenz Brinkmann " occupe le terrain » de façon spectaculaire.

Les recherches sur la "réception" des sculptures antiques dans le monde occidental moderne et contemporain, et sur l'histoire de la discipline, 
sont abordées selon des bases renouvelées qui mettent en cause plus radicalement nos modes de compréhension de la sculpture antique. De nouvelles perspectives sont également envisagées autour de la destination et de la réception des œuvres, concernant en particulier la compréhension et l'appropriation des portraits grecs par les Romains, qui mettent en jeu les liens entre sculpture et histoire sociale et religieuse. La signification sociale des reliefs funéraires continue à susciter la réflexion, tout comme la notion de statue de culte sur le plan religieux : le livre de Gabriele Nick sur L'Athéna Parthénos de l'Acropole a, en particulier, relancé le débat ${ }^{1}$. Ces exemples montrent le poids du modèle athénien dans ce type d'étude: I'enjeu des prochaines années est, sans doute, d'essayer de l'atténuer. Enfin, les relations entre sculpture grecque et civilisations "périphériques" sont vues tantôt sous l'aspect des modèles proche-orientaux et égyptiens, dans l'ombre du livre Black Athena ${ }^{2}$ - un ouvrage dont les répercussions ont été limitées en France -, qui cherchait à mettre en valeur l'héritage africain, particulièrement égyptien, dont la civilisation grecque était redevable, tantôt sous celui des sculptures en marbre du monde phénicien et punique. Les chercheurs de langue allemande ont été particulièrement productifs dans ce dernier domaine, qu'il s'agisse des sarcophages dits anthropoïdes, sculptés pour la plupart en marbre de Paros, ou de l'ensemble mis au jour (essentiellement par l'archéologue français Maurice Dunand) dans le sanctuaire d'Echmoun, près de Sidon ${ }^{3}$. L'œuvre la plus mystérieuse reste, cependant, près de trente ans après sa découverte, la grande statue masculine trouvée à Mozia, ville punique de Sicile orientale, dont la date précise est difficile à établir (vers 470 ou après le milieu du ve siècle avant J.-C. ?) et dont la signification a donné lieu à des dizaines d'articles, sans $q u^{\prime} u n e$ solution vraiment satisfaisante s'impose ${ }^{4}$.

Il est clair que l'on ne peut plus écrire l'histoire de la sculpture grecque commelefaisait/ean Charbonneauxilya quarante ans :les deux volumes publiés dans les années 1990 par Claude Rolley l'ont bien montré5. Des synthèses prenant en compte les nouvelles découvertes et orientations scientifiques verront certainement le jour, en France et à l'étranger, au cours de la prochaine décennie [Antoine Hermary].

Antoine Hermary. Quelssontlesdomaines danslesquelsles découvertes archéologiques des quinze à vingt dernières années ont le plus apporté à la connaissance de la sculpture grecque : chronologie, styles régionaux, attributions aux artistes, autres?

Reinhard Senff. De récentes découvertes archéologiques dans le domaine de la sculpture grecque antique ont généré des résultats significatifs quant à notre compréhension des styles régionaux, non seulement sur le continent grec, sur les îles de la mer Égée et dans la sphère culturelle grecque orientale, mais aussi dans les secteurs géographiques et culturels dépendants comme Chypre. En outre, les nouvelles technologies ont permis de renouveler l'étude scientifique des œuvres, produisant ainsi un nombre important de données qui viennent modifier de manière fondamentale le regard traditionnel porté sur la sculpture grecque. 
1. Kouros dit « de la Porte Sacrée », vers 600 avant J.-C., Athènes, Musée archéologique du Céramique.

2. Kouros dit "de New York" New York, The Metropolitan Museum of Art.

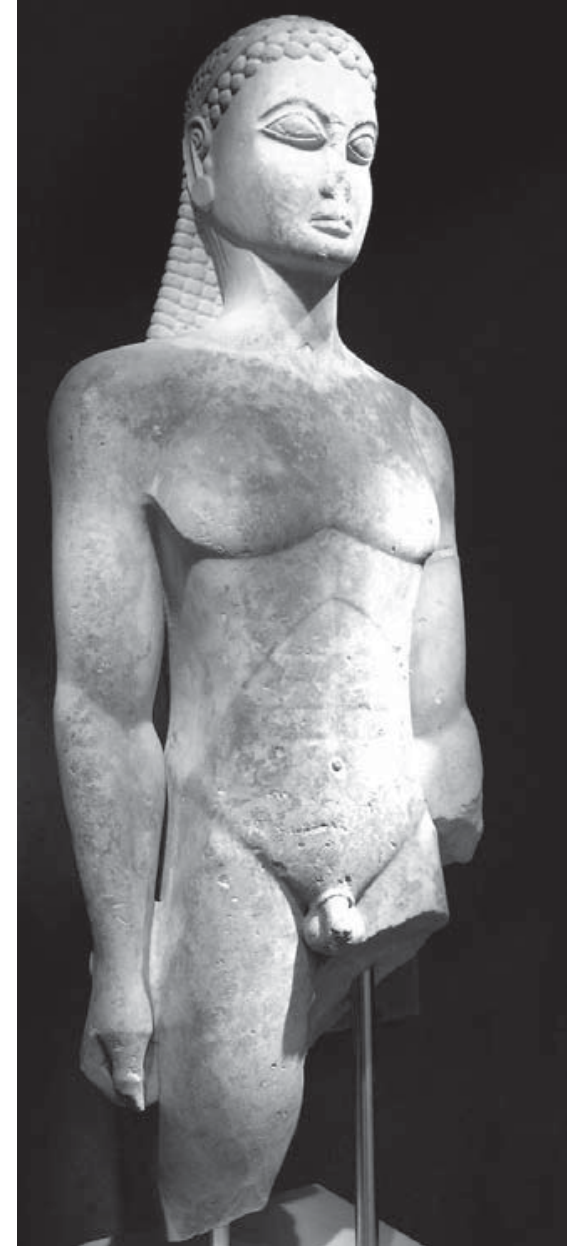

Une des découvertes récentes les plus spectaculaires dans la sphère de la sculpture grecque continentale a été faite par le Deutsches Archäologisches Institut (DAI) au cours de fouilles entreprises au Dipylon d'Athènes, l'entrée principale de la cité par l'ouest. C'est à cet endroit que la voie sacrée d'Éleusis pénétrait dans la ville et que le cortège des Grandes Panathénées se tenait tous les quatre ans. Site majeur faisant face à la porte du Dipylon, lieu de passage particulièrement fréquenté, le célèbre Kerameikos - ou cimetière du Céramique - rassemble de nombreux monuments funéraires de différents types. La plupart des tombes, richement décorées, appartenaient à des familles aisées de l'aristocratie athénienne; il a même été récemment suggéré que I'un des tumuli recouvrait une stèle du début du $\mathrm{Vle}^{\mathrm{e}}$ siècle avant J.-C., qui marquait la tombe d'un important politicien de l'influente famille des Alcméonides. La décoration de la plupart des tombes de la période archaïque a brutalement été détruite lors de la conquête de la cité d'Athènes par les Perses en 480 avant J.-C. Après le retrait des Perses, la démocratie s'affirma comme régime politique à Athènes; il n'y avait alors plus aucune raison de reconstituer les monuments dévolus aux aristocrates. Ainsi, un grand nombre de sculptures mutilées a été utilisé comme matériau de construction pour la nouvelle muraille. Au cours des fouilles menées en 2002 dans le cimetière du Céramique, des sculptures en marbre de grande taille, en très bon état de conservation, ont été retrouvées. Un lion, un sphinx et un kouros grandeur nature, types caractéristiques du contexte funéraire, ont été exhumés non loin de ce qui était peut-être leur emplacement d'origine, dans le cimetière. Le kouros, dit " de la Porte Sacrée ", s'est avéré être une des toutes premières figures monumentales produites dans un atelier attique autour de 600 avant J.-C. (Athènes, Musée archéologique du Céramique ; fig. 1$)^{6}$. II se rapproche étroitement $d^{\prime}$ 'un groupe de sculptures attiques, comprenant une statue entière, le fameux kouros dit « de New York » (New York, The Metropolitan Museum of Art ; fig. 2), ainsi qu'une tête et une main en marbre d'une très grande finesse découvertes lors de fouilles antérieures réalisées dans le même secteur.

Les projets de recherche à long terme, dont un est porté par les universités de Munich et de Bochum en Allemagne, concernent la polychromie de la sculpture et de I'architecture grecques antiques. Leurs résultats, des plus stupéfiants, ont été présentés au public pour la première fois lors de l'exposition retentissante Gods in color, organisée dans différentes capitales européennes et aux États-Unis ${ }^{7}$. La mise en œuvre de nouvelles technologies, comme la microscopie électronique à balayage (MEB) et la photographie fluorescente non destructive, révèlent sur les surfaces sculptées des traces de peinture invisibles à I'œil nu. Les analyses au microscope et le traitement d'échantillons rendent possible l'identification de pigments utilisés dans l'Antiquité. Les résultats modifient de manière significative notre compréhension de la sculpture antique en pierre. Certes, il est depuis longtemps reconnu que les terres cuites de l'époque hellénistique, à l'exemple des fameuses Tanagra, étaient couvertes à l'origine de couleurs éclatantes, mais l'œil moderne, conditionné dans sa perception faussée d'une sculpture en marbre blanc, tend à être choqué par l'apparition de korai archaïques ou de statues classiques et hellénistiques aux draperies éclatantes 
et colorées, aux yeux et aux cheveux peints et à la peau traitée avec réalisme (fig. 3). Tant de détails étaient souvent uniquement rendus au moyen de la couleur sur la surface en pierre! Ces nouveaux résultats montrent combien notre connaissance de la sculpture antique a jusqu'à présent été fragmentaire. Toute analyse future devra les prendre en considération.

Emmanuel Voutiras. Pour faire le point sur la situation actuelle des études sur la sculpture grecque, il est nécessaire de rappeler deux faits qui marquèrent successivement la recherche. II faut tout d'abord garder à l'esprit que le mode de transmission qui caractérise notre connaissance des chefs-d'œuvre de la sculpture classique fut longtemps indirect, passant par les écrits des auteurs anciens et par les copies plus ou moins

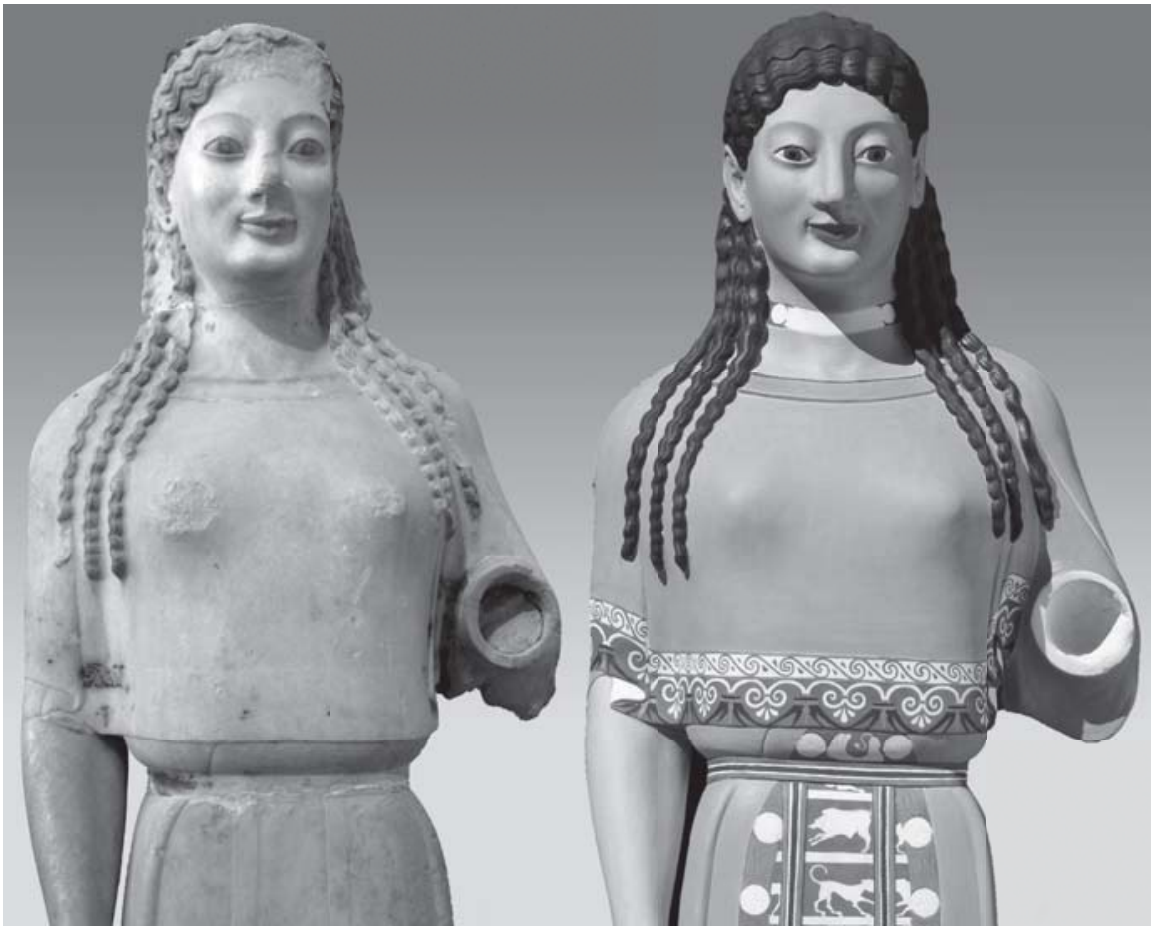
fidèles qui datent pour la plupart de l'époque impériale. Il faut ensuite évoquer les résultats des grandes fouilles menées surtout dans le dernier quart du XIXe et au début du $X X^{e}$ siècle, qui modifièrent profondément la conception des styles et des formes, fondée jusqu'alors essentiellement sur l'étude des copies. Les fouilles de l'Acropole d'Athènes et de Delphes mirent ainsi au jour un nombre considérable de monuments sculptés de l'époque archaïque, permettant d'établir une chronologie fiable et de distinguer des traits stylistiques régionaux, tandis que celles d'Olympie, avec les sculptures du temple de Zeus, firent connaître l'ensemble le plus complet d'œuvres originales du «style sévère ». Les fouilles ont en outre permis de mettre en valeur un matériel aussi vaste que varié. Aussi l'étude de la chronologie et des styles régionaux de la sculpture des époques archaïque et classique s'est-elle fondée non seulement sur les statues et les reliefs de grande taille, mais également sur des œuvres plus modestes mais beaucoup plus nombreuses, comme les statuettes en bronze, sans parler des terres cuites. Les premiers ouvrages solides dans ce domaine, utiles encore aujourd'hui, furent rédigés entre 1920 et $1940^{8}$; ces travaux de base ont permis des pas décisifs dans la connaissance de la sculpture grecque.

Aujourd'hui, les études exclusivement consacrées aux questions de chronologie et de style se font de plus en plus rares. La raison en est sans doute que la méthode a peu évolué, ce qui n'exclut pourtant pas d'intéressantes tentatives pour mettre en évidence la spécificité de la production artistique d'une région ${ }^{9}$. Il arrive aussi que des trouvailles exceptionnelles permettent de rouvrir de vieux dossiers, comme le montre la récente découverte à Athènes, près du Dipylon, d'un ensemble important de statues funéraires de l'époque archaïque, déjà évoqué par Reinhard Senff. Un domaine dans lequel notre savoir a avancé grâce, entre autre, à des trouvailles récentes est celui des techniques de fabrication. Ainsi la mise au jour des statues de Riace a donné une impulsion à la recherche sur la fonte des grands bronzes à la cire perdue ${ }^{10}$. Dans un autre domaine, les observations précises de Giorgos Despinis ont permis de comprendre la technique des statues acrolithes ${ }^{11}$.
3. Koré péplophore : a. 530-520 avant J.-C., Athènes, Musée de I'Acropole ; b. restitution partielle du décor polychrome, variante $\mathrm{C}$, Vinzenz Brinkmann, Ulrike Koch-Brinkmann, Heinrich Piening [Bunte Götter: die Farbigkeit antiker Skulptur, Vinzenz Brinkmann, Raimund Wünsche éd., (cat. expo., Munich, Staatlichen Antikensammlungen und Glypothek, 2003-2004), Munich, 2004, fig. 83].

4. Restitution du fronton est du dernier temple d'Apollon, IV siècle avant J.-C., sanctuaire de Delphes, frontons du temple du IV siècle, Athènes, 2003, pl. 87].
Grèce [Francis Croissant, Les

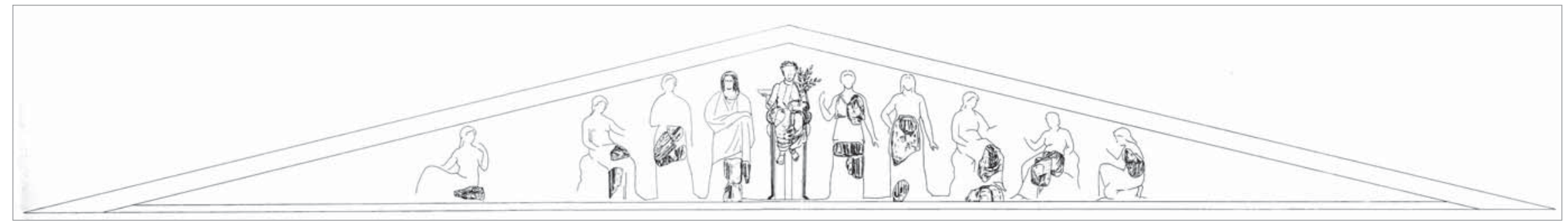



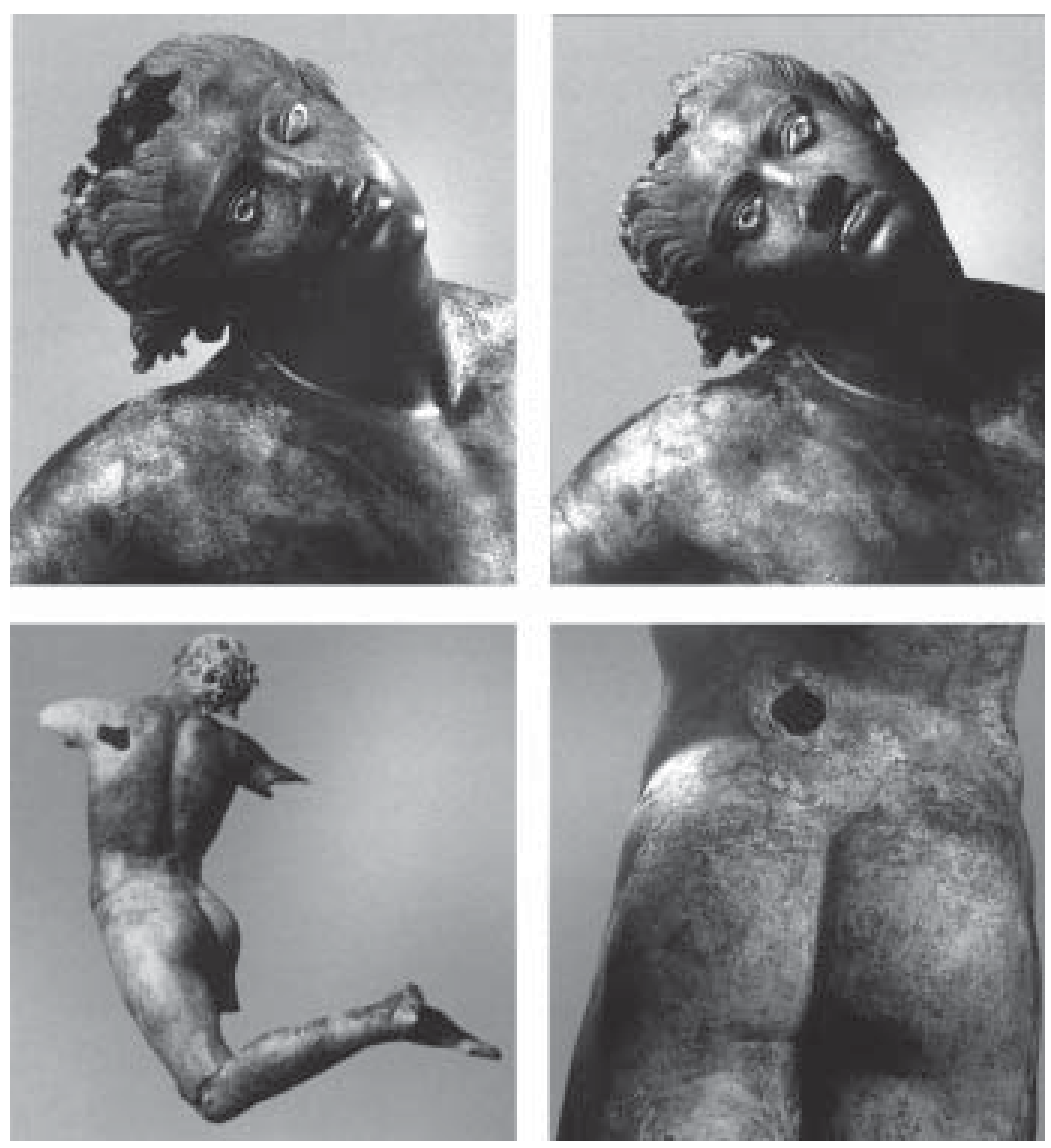

La mise en valeur de pièces insuffisamment étudiées ou méconnues a été à l'origine de quelques belles découvertes. Ainsi un travail assidu dans les réserves du musée de Delphes a permis de restituer les compositions des deux frontons du temple d'Apollon du IV siècle avant J.-C., que I'on croyait depuis longtemps perdus (fig. 4) ${ }^{12}$. Une tête féminine colossale en marbre de Paros du Musée de l'Acropole d'Athènes, une fois sa vraie provenance établie, a pu être identifiée avec beaucoup de vraisemblance comme celle d'Artémis Brauronia, mentionnée par Pausanias dans sa description de l'Acropole comme étant une œuvre de Praxitèle ${ }^{13}$. Cette découverte oblige à reconsidérer certaines idées reçues sur la production artistique du célèbre sculpteur, comme l'a montré l'exposition récemment organisée au Louvre ${ }^{14}$.

Irene Bald Romano. Les considérations sur la sculpture grecque ont été radicalement affectées au cours de ces vingt dernières années, notamment par de nouvelles approches méthodologiques, théoriques et scientifiques, et dans une moindre mesure par des découvertes archéologiques. On compte

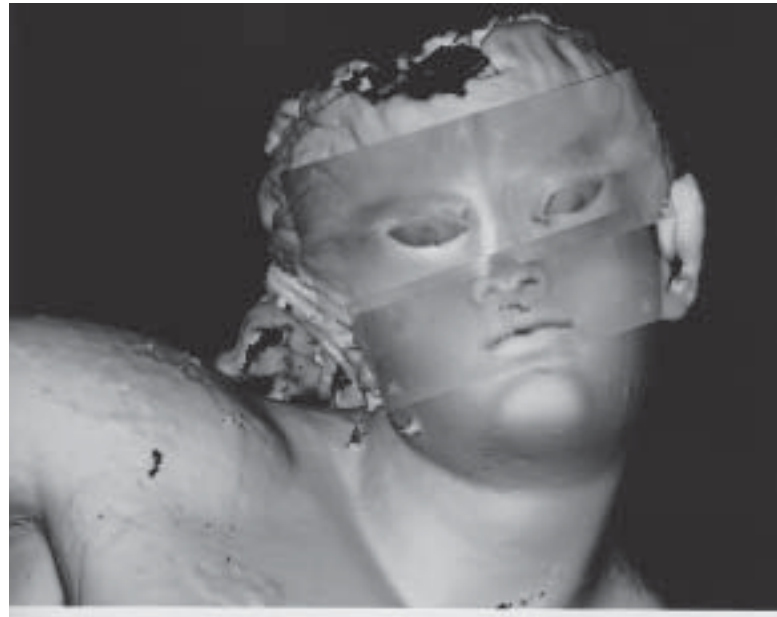
toutefois des découvertes extraordinaires depuis vingt-cinq ans, en particulier des bronzes révélés par l'archéologie. La paire de guerriers du ve siècle avant J.-C., trouvée en 1972 au large de la côte de Riace, près de Reggio de Calabre (Reggio de Calabre, Museo nazionale della Magna Grecia), a nourri de nombreuses publications ${ }^{15}$. Une cachette de bronzes trouvée en 1992 près de Brindisi ${ }^{16}$, dont les fragments couvrent une période de plusieurs centaines d'années, du II siècle av. J.-C. à l'époque impériale avancée, comprenait, entre autres, l'image d'un "souverain hellénistique ${ }^{17}$. Le Satyre dansant trouvé au large de la côte du sud-ouest de la Sicile près de Mazara del Vallo en 1998 (Museo del Satiro, Mazara del Vallo; fig. 5a), dont le style n'est pas sans rappeler celui de Praxitèle, a ainsi renouvelé l'intérêt et le débat sur l'œuvre de ce sculpteur ${ }^{18}$. Enfin, un Apoxymène, probablement de la fin de l'époque hellénistique, a été retrouvé au large de l'île croate de Lošinj en 1999 (Zagreb, Musée archéologique). Dans tous les cas, ces découvertes ont donné lieu à d'importantes campagnes de restauration, à des analyses techniques et, pour la plupart, à des publications conséquentes venues enrichir notre connaissance des techniques et des usages de la sculpture antique en bronze (fig. 5b) ${ }^{19}$.

Dans le domaine de la sculpture en marbre, des découvertes remarquables ont été faites, à l'exemple du kouros de presque cinq mètres de haut offert par un notable nommé Ischès, trouvé en plusieurs étapes - 1974, 1981 et 1984 - dans le sanctuaire de Héra à Samos (Samos, MuséearchéologiquedeVathy) ${ }^{20}$; etun second kouros, ressemblantétroitementàcelui du Dipylon, a été découvert en 2002 près de la Porte Sacrée dans le cimetière du Céramique, avec un sphinx, deux lions en marbre et les fragments de deux stèles en marbre ${ }^{21}$. Le kouros en marbre thasien acquis par le Getty Museum à Malibu dans les années 1980, ce qui lui a valu sa réputation, a peut-être généré plus d'intérêt que les fouilles archéologiques elles-mêmes. À l'instar des sculptures en bronze issues de fouilles subaquatiques, il a été soumis à tous les examens possibles en matière de science et d'analyse scientifique. Reste que, finalement, son authenticité est loin de faire l'unanimité auprès des chercheurs. Le Getty Museum lui-même l'étiquette comme « grec, environ 530 avant J.-C., ou contrefaçon moderne ».

5. Satyre dansant, IV ${ }^{\mathrm{s}}$ siècle avant J.-C. ou production néo-attique, Mazara del Vallo, Museo del Satiro : a. prises de vues pendant la campagne de restauration; b. radiographies sur vue numérisée en 3D. 
De fait, les questions de l'acquisition illégale et immorale d'œuvres d'art grec, comprenant la sculpture, et la tentative de vérifier la légitimité des propriétaires tout comme l'authenticité des œuvres ont conduit la communauté scientifique à être dorénavant plus attentive aux techniques, à l'utilisation des matériaux et aux styles régionaux. J'en veux pour preuve une autre pièce bien connue acquise par le Getty Museum en 1988, une statue colossale de déesse, peut-être Aphrodite, datant de la fin du ve siècle avant J.-C., qui a fourni des informations quant à l'utilisation de la technique pseudo-acrolithe (dotée d'un corps en calcaire, alors que la tête, les bras et les pieds sont en marbre parien) et sa portée régionale. Lors d'une journée d'études organisée au Getty Museum en mai 2007, Clemente Marconi a montré que, à l'époque classique, la Sicile est la seule région qui puisse revendiquer cette technique, son utilisation étant suscitée par le manque de marbre dans la région ${ }^{22}$. La statue, probablement issue d'un pillage sur le site de Morgantina en Sicile, sera rapatriée en Italie en 2010, selon un accord passé entre le Getty Museum et le Ministère italien de la culture. De même, la récente inauguration du Musée de l'Acropole, dont la muséographie permet de lire les sculptures selon un nouvel éclairage - aux deux sens du terme -, nous invite à porter un autre regard sur ces œuvres : cet écrin spectaculaire dévolu aux sculptures de l'Acropole va certainement intensifier le débat sur le retour des marbres du Parthénon conservés au British Museum.

Du point de vue de la méthode, l'étude de la sculpture grecque antique a été transformée par l'historiographie et l'histoire de la réception. Ces deux approches ont donné lieu à des études contemporaines des plus stimulantes dans le domaine de l'art antique. En ce qui concerne I'historiographie, Greek Sculpture and the Problem of Description d'Alice Donohue (Cambridge/New York, 2005) présente les tendances et les facteurs intellectuels, culturels et historiques qui ont marqué les études de la sculpture grecque au cours des trois dernières décennies. Un essai antérieur édité par Alice Donohue et Mark Fullerton, Ancient Art and its Historiography (Cambridge/New York 2003), mettait déjà en évidence ce type d'approche avec d'importantes contributions de divers chercheurs. Ces études ont encouragé les spécialistes de la sculpture grecque à être prudent vis-à-vis d'hypothèses excessives - notamment en ce qui concerne les études stylistiques - et à accepter les limites de notre capacité actuelle à comprendre et à lire la culture visuelle grecque antique. Dans cette optique, Art, Desire, and the Body in Ancient Greece d'Andrew Stewart (Cambridge, 1997) est un essai révolutionnaire en ce qu'il examine la conception grecque de la nudité, montrant en quoi elle nous éclaire sur l'intellect et la manière dont pensait la société dans l'Antiquité grecque, particulièrement en ce qui concerne le genre et la sexualité.

Les études scientifiques mentionnées ci-dessus ont aussi ouvert le champ des recherches sur la sculpture grecque à de nouveaux horizons. Au cours des vingt dernières années, les travaux collectifs d'historiens de l'art, d'archéologues, de conservateurs, de chimistes et de géologues sur les marbres antiques ont fourni des informations essentielles sur les techniques. L'analyse des isotopes stables, par exemple, a permis d'identifier les marbres, de déterminer l'utilisation et la chronologie des carrières antiques, le commerce du marbre et le symbolisme de l'utilisation de certaines pierres. Le mérite de cette collaboration internationale exceptionnelle revient en majeure partie à l'audacieux géologue Norman Herz, professeur émérite à I'University of Georgia, instigateur et moteur de la formation d'ASMOSIA en 1988 (fig. 6). Les actes des huit colloques internationaux organisés par cette Association pour l'étude des marbres et autres roches utilisés dans le passé constituent les sources principales d'informations sur le sujet.

6. Site Internet d'ASMOSIA : page d'accueil (www.asmosia.org).

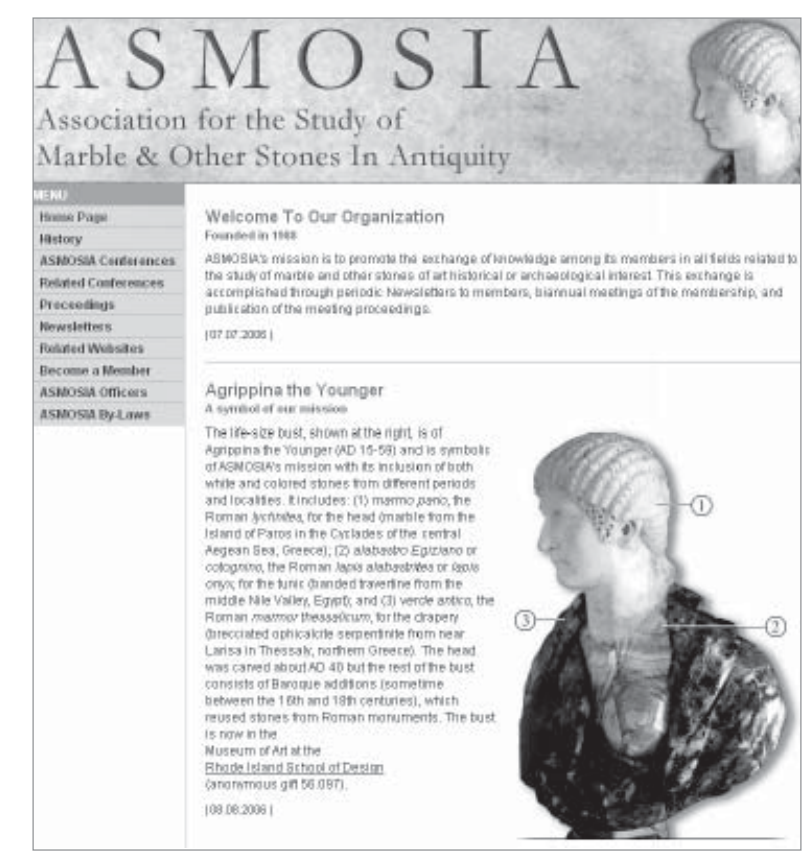


Enfin, I'utilisation des techniques photographiques et scientifiques pour identifier la peinture et d'autres substances appliquées sur les surfaces de la sculpture grecque en marbre - par exemple, dorures ou patines artificielles - a changé notre regard sur la sculpture grecque antique. D'importants articles ont été publiés à ce sujet dans les actes des colloques organisés par ASMOSIA, dont ceux de Brigitte Bourgeois et Philippe Jockey sur la sculpture de Délos ${ }^{23}$. Mais le travail le plus influent réalisé sur la polychromie de la sculpture grecque a été effectué par Vinzenz Brinkmann et une équipe internationale de la Liebieghaus Skulpturensammlung à Francfort. Des expositions de grande ampleur accompagnées de catalogues ont été présentées en Europe et aux États-Unis au cours des cinq dernières années, dont la dernière en date, Gods in Color, a déjà été citée. Si l'importance de la théorie de la réception et de I'historiographie ne fait aucun doute, nous reconnaissons désormais que la recherche des siècles précédents a déformé notre regard sur sculpture grecque antique. La première sculpture grecque en marbre n'était pas immaculée, mais au contraire richement colorée; les sources antiques l'attestent. La recherche antérieure a nié l'évidence parce qu'une telle conception ne s'accordait pas avec la vision esthétique de sa propre époque.

Antoine Hermary. Quelle est actuellement la contribution de la sculpture aux études sur l'histoire sociale et religieuse du monde grec antique?

Emmanuel Voutiras. L'art grec a longtemps été considéré et étudié dans une perspective classiciste visant surtout à mettre en valeur ses mérites esthétiques. La sculpture en particulier a joué un rôle prépondérant dans cette perception et cette appréciation, fermement établie bien avant l'apparition de l'archéologie comme discipline indépendante. Depuis la Renaissance, I'admiration des artistes, des connaisseurs et des érudits pour la statuaire antique, et en particulier grecque, n'a cessé de s'affirmer. Il suffit de rappeler la sensation provoquée par la découverte à Rome, au début du XVI siècle, de deux créations remarquables de la sculpture hellénistique, le groupe de Laocoon et le Torse du Belvédère ${ }^{24}$, qui exercèrent une influence considérable sur l'œuvre d'artistes tels que Michel-Ange.

La recherche actuelle n'est plus axée sur la valeur artistique de la sculpture grecque, mais tend plutôt à exploiter le témoignage qu'elle apporte sur la culture et la société de la Grèce antique. Cette orientation s'est illustrée dans des efforts pour restituer l'aspect original des œuvres et les situer dans leur contexte d'origine. Un bel exemple est donné par la reconstitution, à l'aide de moulages, de la statue de Ménandre érigée par les Athéniens dans le théâtre de Dionysos au début du II $^{\mathrm{e}}$ siècle avant J.-C., peu après la mort du célèbre poète comique ${ }^{25}$.

L'analyse systématique de l'iconographie et la remise en contexte archéologique des monuments sculptés ont également permis d'en tirer parti pour la connaissance de la religion grecque. C'est dans cette perspective que se situe une tentative récente de définir la fonction de ce qu'il est convenu d'appeler une «statue de culte ${ }^{26}$. L'étude typologique et iconographique des sculptures intégrées dans un édifice sacré révèle que certaines figures généralement considérées comme décoratives avaient en réalité une fonction cultuelle. Ainsi les Caryatides de l'Érechthéion sur l'Acropole d'Athènes ont été récemment interprétées comme des "choéphores», des jeunes filles apportant des libations à la tombe de Kékrops ${ }^{27}$. De même, l'étude très poussée de l'iconographie et des caractéristiques techniques d'un beau relief provenant du sanctuaire d'Artémis à Brauron en Attique, que I'on peut dater de la décennie 420-410 avant J.-C., a mis en évidence le rapport étroit de cette œuvre avec l'introduction du culte d'Iphigénie, évoqué dans I'Iphigénie en Tauride d'Euripide, produite à Athènes dans ces mêmes années ${ }^{28}$. 
Certaines tentatives de lire la sculpture grecque dans l'optique d'une histoire sociale sont en revanche moins convaincantes. La commande de statues et de reliefs, soit comme dédicaces, soit comme monuments funéraires, était indubitablement un privilège des classes aisées, mais il n'est pas facile de déterminer le statut social des commanditaires à partir de la facture ou de l'iconographie de ces monuments. Une étude récente replace les stèles et les monuments funéraires attiques de la fin du ve et du IV siècle avant J.-C. dans une perspective sociologique (fig. 7) : les images familiales, souvent présentes sur ces monuments, seraient une expression symbolique du pacte familial (oikos) dans ses rapports avec la cité (polis) ${ }^{29}$. Il faut cependant remarquer que cette interprétation, fondée sur des données quantitatives, réduit la représentation des défunts et de leurs familles à des images conventionnelles et masque leur complexité.

Irene Bald Romano. Le portrait grec a été, jusqu'à récemment, un sujet largement confiné à l'identification des personnages et des copies romaines (la traditionnelle Kopienkritik). Cependant, de nouvelles recherches explorent une autre dimension dans le but de replacer ces portraits dans leur contexte social et politique, tant grec que romain. À cet égard, trois publications se distinguent: Hellenistic Royal Portraits de Roland Smith (Oxford, 1988), The Mask of Socrates: The Images of the Intellectual in Antiquity de Paul Zanker (Berkeley, 1996) et Ancient Greek Portrait Sculpture: Contexts, Subjects, and Styles de Sheila Dillon (New York, 2006). Dans ces trois ouvrages, la réflexion s'articule autour des mêmes questions : pour qui étaient réalisés ces portraits ? Comment étaientils utilisés et considérés? Que signifiaient-ils? Smith déchiffre I'histoire politique complexe du monde hellénistique et la place qui y était accordée aux portraits. Le propos de Zanker est centré sur l'image mouvante des grands intellectuels, tels que les philosophes et les poètes, omniprésents dans l'histoire culturelle gréco-romaine, et se réfère, comme figure de comparaison, au milieu intellectuel allemand du XIXe siècle. Dillon traite des questions telles que la vision des Grecs ou l'interprétation romaine (I'auto-conceptualisation grecque et la réinterprétation romaine de celle-ci) ; comment et pourquoi les artisans romains ont-ils peint les Grecs de telle ou telle manière ; l'exposition de ces portraits de Grecs dans les galeries d'art antiques et dans la sphère tant publique que privée du monde romain ; et leur réception auprès du public romain. Ces ouvrages ont conduit à moduler le discours scientifique contemporain, dans des directions qui mettent davantage en lumière l'histoire culturelle et sociale de la Grèce antique et de Rome.

Les études iconographiques qui ont recours à la sculpture grecque pour éclairer les différents aspects de I'histoire de la religion grecque ont incommensurablement été aidées, depuis les années 1980, par le méthodique Lexicon iconographicum mythologiæ classicæ (LIMC), une publication achevée en 1999, mais enrichie par un supplément publié en 2009 et, depuis 2005, disponible sur Internet ${ }^{30}$. Ce travail a été complété par le Thesaurus Cultus et Rituum Antiquorum (ThesCRA), exclusivement centré sur le domaine cultuel et rituel ${ }^{31}$. Peu d'autres outils ont eu un tel effet sur les études de l'iconographie gréco-romaine antique, rendant ainsi possible des recherches importantes qui s'appuient sur des images de la sculpture grecque, à I'exemple de l'ouvrage de Joan Breton Connelly sur les prêtresses grecques et sur le rôle clé des femmes dans le domaine des cultes grecs antiques et dans la vie religieuse (fig. 8) ${ }^{32}$.

8. Reliefs funéraires consacrés à des prêtresses, Athènes, Musée archéologique national et Berlin, Staatliche Museen Antikensammlung [Joan Breton Connelly, Portrait of a Priestess: Women and Ritual in Ancient Greece, Princeton/Oxford, 2007, p. 239].

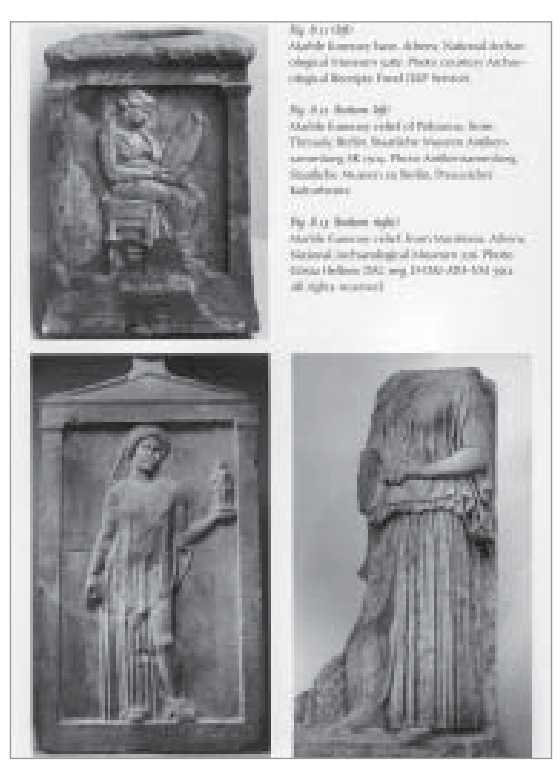


La vue athénocentrique de la sculpture grecque a cédé la place, depuis vingt ans, à l'étude de corpus de sculptures d'autres régions, qui vise à dégager les caractéristiques régionales et la manière dont ces dernières reflètent des attitudes artistiques, sociales, politiques et religieuses, ou encore le caractère d'une cité ou d'une région ${ }^{33}$. L'exposition récente organisée à l'Onassis Cultural Center de New York, Athens-Sparta, a mis en œuvre une approche comparative de l'étude de la production artistique des deux cités-États grecques - Athènes et Sparte à travers la sculpture, parmi d'autres catégories d'objets, pour examiner comment leurs propres histoires, leur idéologie politique et leurs normes culturelles ont influé sur la production artistique à diverses époques ${ }^{34}$. Pour la Laconie, par exemple, peu de kouroi ou de korai, les types idéaux de l'Athènes aristocratique à la période archaïque, sont documentés. Sparte, en revanche, avait une forte préférence, dès le milieu du Vle siècle avant J.-C. et jusqu'à la période romaine, pour les bas-reliefs honorant des ancêtres héroïsés mythiques, aussi bien masculins que féminins, comme les Dioscures ou des divinités chthoniennes. Il existe des interprétations contradictoires des bas-reliefs de héros laconiens, quant à leur utilisation comme marqueurs de tombe ou de lieux sanctifiés ou légendaires, et quant à leur aspect mémoriel, ceux-ci ne se rapportant pas à l'individu mais au genos spartiate ${ }^{35}$.

Reinhard Senff. En effet, les recherches actuelles ne sont plus centrées sur des cas isolés et ne visent plus uniquement à rattacher une œuvre à un sculpteur célèbre ou à un atelier régional. C'est au contraire la remise en contexte des œuvres d'art qui importe désormais. Par exemple, à l'examen de la statuaire funéraire antique - stèles, objets en céramique, statues -, la gestuelle des figures humaines est interprétée dans le respect du message qu'elle délivre au spectateur, y compris politique ${ }^{36}$. De même, les différents types de sculptures votives découvertes dans un sanctuaire - qu'il s'agisse de l'Acropole d'Athènes ou d'une cité secondaire - sont appréhendés et mis en regard avec d'autres sortes d'offrandes votives. Ils témoignent ainsi des diverses catégories de la société et des objets de cultes utilisés lors des célébrations ${ }^{37}$.

Antoine Hermary. Les découvertes et les recherches récentes ont-elles modifié notre vision des rapports entre la sculpture grecque et celle des civilisations " périphériques " (Anatolie, Phénicie, Chypre, Étrurie, monde ibérique)?

Irene Bald Romano. Les origines de l'art grec, en particulier la sculpture grecque et ses liens avec l'Égypte et le Proche-Orient, ont été I'un des sujets les plus stimulants des vingt dernières années, à la fois pour la communauté scientifique et, dans une certaine mesure, pour un plus large public. Je pense notamment aux volumes controversés de Black Athena, dans lesquels Martin Bernal revendique les origines africaines de la civilisation grecque ${ }^{38}$. Une autre évaluation scientifique récente de Bernal, qui porte sur ce qui est égyptien dans la première sculpture grecque, est au centre de la contribution de Jeremy Tanner dans les actes du colloque Ancient Perspectives on Egypt (2003) ${ }^{39}$.

Il existe un vaste ensemble de preuves des interconnexions entre la Grèce, le Proche-Orient et l'Égypte dès l'âge du bronze via la mer Égée ${ }^{40}$, et certainement autour du $\mathrm{VIII}^{\mathrm{e}}$ siècle avant J.-C. et plus tard encore. La période comprise entre les $\mathrm{XI}^{\mathrm{e}}$ et IX $\mathrm{X}^{\mathrm{e}}$ siècles avant J.-C. est plus obscure, mais la tendance générale est d'assumer l'idée d'une persistance des contacts et d'un renouvellement ou d'un renforcement de ceux-ci entre la Méditerranée orientale et le Proche-Orient autour du IX siècle, par l'intermédiaire des Phéniciens. Pour la sculpture grecque, il semble clair que l'engouement au VIII siècle avant J.-C. pour la création d'images de culte en tant que substituts anthropomorphiques des divinités, dans les sanctuaires grecs et les temples aussi bien que les pratiques de culte 
associées à ces images, est dû aux influences de l'Égypte ${ }^{41}$. En utilisant une approche historiographique et en combinant les témoignages littéraires, historiques et archéologiques, Sarah P. Morris expose les motifs de l'influence profonde du Proche-Orient sur le premier art grec, y compris la sculpture, dans Daidalos and the Origins of Greek Art (Princeton, 1992). Morris étant sur les traces du Dédale mythique, elle sépare le Dédale littéraire de la première sculpture grecque et recommande l'abrogation du terme dédalique pour décrire le style sculptural du VII siècle avant J.-C., celui-ci n'étant pas représentatif du large éventail de styles régionaux appliqués à des médiums variés, à une période qui devrait plus exactement être qualifiée $d^{\prime}$ " orientalisante ». Un livre à paraître de Jane Carter sur les débuts de l'art grec portera plus loin ces questions sur la part orientale de la sculpture grecque.

Reinhard Senff. Des découvertes spectaculaires ont été faites en Grèce orientale. La sphère culturelle d'Ionie qui, selon la tradition, a été colonisée depuis le continent à la fin du $\|^{\mathrm{e}}$ et au début du $I^{\text {er }}$ millénaire avant J.-C., a rapidement développé une identité culturelle propre ainsi qu'un haut niveau artistique. À Phocée, cité-mère de la colonisation grecque en Méditerranée occidentale et de Massalia (actuelle Marseille), de récentes fouilles ont permis de mettre au jour un temple archaïque décoré de grandes protomés de chevaux et de griffons qui dénotent une extraordinaire maîtrise technique et manifestent, encore une fois, l'originalité de la Grèce orientale et la haute qualité de ses objets. Plus au sud, à Milet, capitale de la Confédération ionienne, ont été récemment faites de nombreuses découvertes sur la sculpture de la période archaïque lors de nouvelles fouilles du sanctuaire d'Aphrodite, aussi célèbre en son temps que celui de Paphos sur l'île de Chypre. Outre les extraordinaires figurines de bronze qui avaient autrefois appartenu à de grands navires et quelques sculptures en marbre typiquement ioniennes, des centaines de figurines archaïques en terre cuite ont été trouvées, représentant des fidèles et la déesse elle-même ; elles illustrent des types iconographiques jusqu'alors inconnus. S'il s'agit clairement de productions d'ateliers locaux, ces œuvres affichent également des caractéristiques venues de l'iconographie proche-orientale et crétoise, et témoignent ainsi des contacts internationaux et de l'ouverture aux influences étrangères, l'Ionie jouant le rôle de passerelle entre l'Orient et l'Occident. D'abondantes importations du Levant et d'Égypte illustrent les itinéraires commerciaux qui, à cette époque, étaient les artères des contacts culturels. Un grand nombre de figurines importées, en terre cuite ou en calcaire chypriote, a récemment été mis au jour non seulement à Milet, mais aussi dans un sanctuaire à Emecik sur la presqu'île de Cnide et dans I'Heraion de Samos (où les découvertes, plus anciennes, furent abondantes) ${ }^{42}$, ce qui atteste que Chypre était bien intégrée dans ce réseau primitif. Le débat scientifique actuel s'attache à caractériser les composantes de ces objets d'art, à distinguer ce qui est proprement grec, chypriote, oriental ou égyptien.

Emmanuel Voutiras. Les débuts de la sculpture monumentale en Grèce sont marqués par des influences provenant du Proche-Orient et de l'Égypte. Cependant, dès l'époque archaïque, et surtout à partir de l'époque classique, la sculpture grecque a, à son tour, fortement marqué l'art des pays de la Méditerranée orientale. L'existence de ces rapports réciproques est unanimement reconnue, mais leur nature n'est pas toujours évidente et elle est constamment remise en question. La situation est sans doute plus claire dans le cas de l'Étrurie, dont la production artistique (et tout particulièrement la sculpture) a profondément et durablement été influencée par l'art grec, aussi bien dans le style que dans le choix des sujets mythologiques ${ }^{43}$.
9. Sarcophage d'homme provenant de Sidon, $2^{\mathrm{e}}$ quart du V viècle avant J.-C., Istanbul, Musée archéologique.

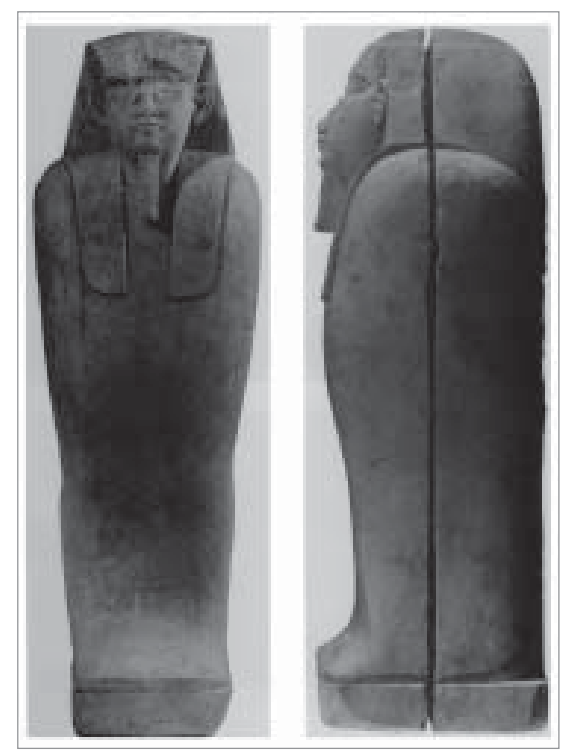




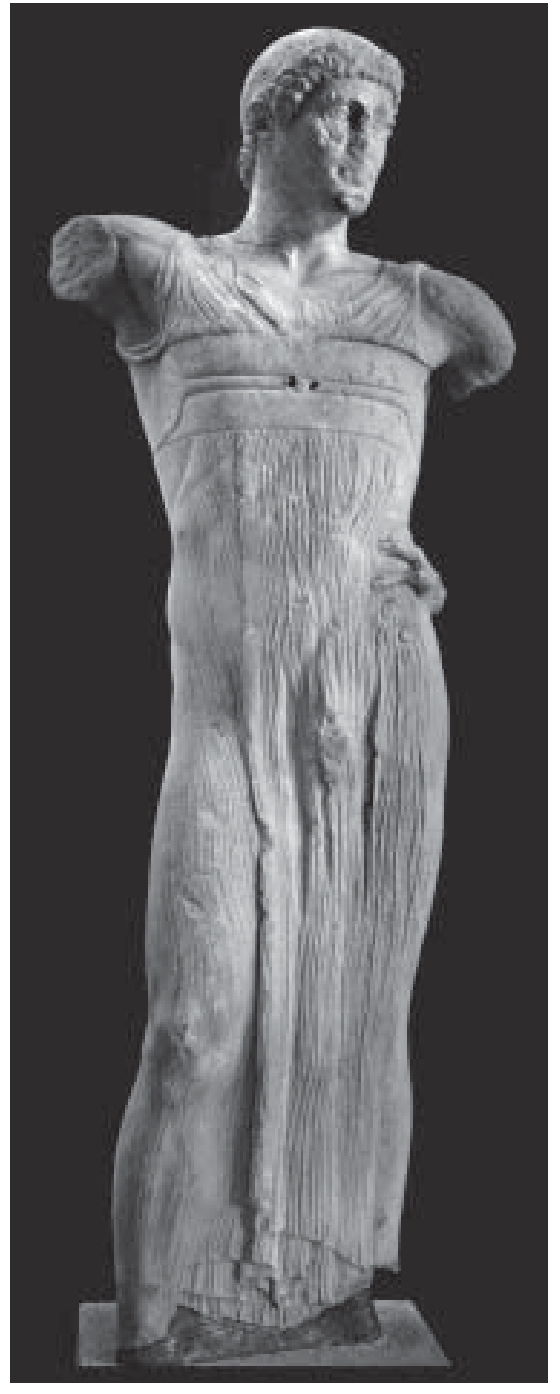

10a. Statue masculine trouvée à Mozia (Sicile orientale), dit L'Aurige ou L'Éphèbe de Mozia, 470-460 avant J.-C., Mozia, Musée archéologique; b. détail.

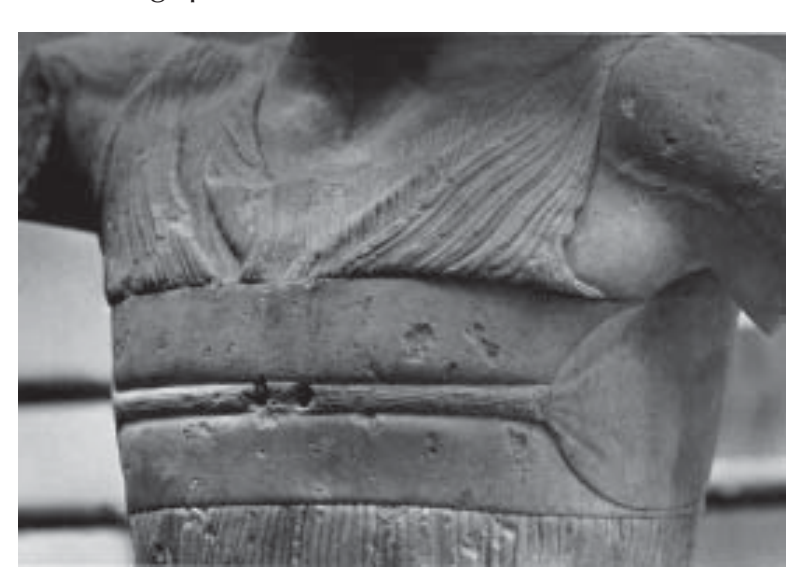

L'influence de la sculpture grecque sur les œuvres des "civilisations périphériques » se manifeste, dans la plupart des cas, à travers la facture et le style. En témoignent les sarcophages anthropoïdes phéniciens, issus d'un alliage du cercueil momiforme égyptien et de la sculpture grecque (fig. 9). Une étude récente dédiée à ces monuments funéraires, qui se retrouvent jusqu'à Carthage et Gadès et dont la production couvre le $\mathrm{V}^{\mathrm{e}}$ et le IV siècle avant J.-C., a mis en évidence les diverses composantes ${ }^{44}$. Le matériau, le marbre de Paros, et le style montrent que les créateurs des premiers exemplaires, mis au jour à Sidon, étaient des Grecs, venus probablement des îles de l'Égée, qui ont ensuite formé des disciples locaux. La présence de sculpteurs grecs est particulièrement évidente à Sidon, dans le sanctuaire d'Echmoun, et dans les sarcophages sculptés de la nécropole royale, parmi lesquels le "sarcophage d'Alexandre " mérite une mention spéciale ${ }^{45}$. Le principal problème est de savoir dans quelle mesure certaines sculptures de style purement grec recèlent des éléments iconographiques phéniciens.

Il faut enfin mentionner dans ce contexte une trouvaille aussi impressionnante qu'énigmatique qui a fait couler beaucoup d'encre depuis sa découverte en 1979 : la statue de Mozia (au musée archéologique local ; fig. 10) ${ }^{46}$. Ce chef-d'œuvre de la sculpture grecque du style sévère, que I'on peut dater d'environ 470-460 avant J.-C., fut trouvé dans l'installation punique sur I'îlot de Mozia (Motyè en grec) dans la lagune de Marsala en Sicile, près d'un sanctuaire. Le personnage représenté est un homme jeune vêtu d'une longue tunique rappelant celle des auriges, attachée à hauteur de la poitrine par une large ceinture. La question fondamentale qui se pose est de savoir si la statue a été conçue pour Mozia ou si elle fut apportée d'une ville grecque de Sicile. Si l'origine grecque du sculpteur est indiscutable, certains éléments du vêtement, à commencer par la ceinture, n'ont pas de parallèle dans l'art grec. La question reste donc ouverte, même si, dans l'état actuel de nos connaissances, il paraît difficile de privilégier l'hypothèse $d$ 'une commande carthaginoise. Il est intéressant de constater comment la sculpture grecque, traditionnellement admirée pour sa valeur esthétique, devient dans la recherche moderne, qui privilégie surtout l'étude des techniques et cherche à placer les œuvres dans leur contexte d'origine, un témoignage de la civilisation grecque et de ses rapports avec les autres civilisations de la Méditerranée antique.

Nota bene : ce texte résulte de l'envoi de questions aux participants et d'un échange de courriels. 
1. Gabriele Nick, Die Athena Parthenos: Studien zum griechischen Kultbild und seiner Rezeption, (MDAI[A], 19), Francfort, 2002.

2. Martin Bernal, Black Athena: The Afroasiatic Roots of Classical Civilization, I, The fabrication of ancient Greece 1785-1985, New Brunswick, 1987 [éd. fr. : Black Athena: les racines afro-asiatiques de la civilisation classique, I, L'invention de la Grèce antique, 1785-1985, Paris, 1996]; II, The archaeological and documentary evidence, New Brunswick, 1991; III, The linguistic evidence, New Brunswick, 2006.

3. Hanns Gabelmann, «Zur Chronologie der Königsnekropole von Sidon », dans Archäologischer Anzeiger, 1979, p. 163-177; Rolf A. Stucky, Die Skulpturen aus dem Eschmun-Heiligtum bei Sidon. Griechische, römische, kyprische und phönizische Statuen und Reliefs vom 6. Jahrhundert vor Chr. bis zum 3. Jahrhundert nach Chr., (Antike Kunst Beiheft, 17), Bâle, 1993.

4. Voir Nicola Bonacasa, Antonino Buttitta éd., La Statua marmorea di Mozia e la scultura di stile severo in Sicilia, (colloque, Marsala, 1986), Rome, 1988.

5. Claude Rolley, La sculpture grecque, I, Des origines au milieu du ve siècle, Paris, 1994; II, La période classique, Paris, 1999; voir aussi les importants travaux de Brunilde Sismondo Ridgway et d'Andrew Stewart.

6. Wolf-Dietrich Niemeier, Der Kuros vom Heiligen Tor: überraschende Neufunde archaischer Skulptur im Kerameikos in Athen, Francfort, 2002.

7. Gods in Color: Painted Sculpture of Classical Antiquity, (cat. expo., Cambridge, Arthur M. Sackler Museum/Francfort, Liebieghaus, 2007-2009), Cambridge (MA), 2007.

8. Il suffit de citer le livre «fondateur» d'Ernst Langlotz, Frühgriechische Bildhauerschulen, Nuremberg, 1927, qui a fait date.

9. Par exemple, Olga Palagia, William Coulson éd., Sculpture from Arcadia and Laconia, (colloque, Athènes, 1992), (Oxbow monograph, 30), Oxford, 1993.

10. Guglielmo B. Triches éd., Due bronzi da Riace: rinvenimento, restauro, analisi ed ipotesi di interpretazione, 2 vol., (Bollettino d'arte, série spéciale, 3), Rome, 1984 ; Alessandra Melucco Vaccaro et al. éd., I bronzi di Riace: restauro come conoscenza, 3 vol., Rome, 2003.

11. Voir en dernier lieu Giorgos Despinis, $Z u$ Akrolithstatuen griechischer und römischer Zeit, (Nachrichten der Akademie der Wissenschaften zu Göttingen, 1/8), Göttingen2004.

12. Francis Croissant, Les frontons du temple du IV siècle, (Fouilles de Delphes IV. Monuments figurés, sculpture, 7), Athènes, 2003.

13. Giorgos Despinis, «Neues zu einem alten Fund ", dans Mitteilungen des Deutschen
Archäologischen Instituts, Athenische Abteilung [MDAI(A)], 109, 1994, p. 173-198.

14. Praxitèle, Alain Pasquier éd., (cat. expo., Paris, Musée du Louvre, 2007), Paris, 2007.

15. Triches, 1984, cité n. 10.

16. Giuseppe Andreassi, Bronzi di Punta del Serrone: ricerche archeologiche subacquee a Brindisi nel 1992, (Bollettino di Archeologia, supplément), Rome, 1992.

17. The Fire of Hephaistos. Large Classical Bronzes from North American Collections, Carol C. Mattusch éd., (cat. expo., Cambridge, Arthur M. Sackler Museum/Tolède, Museum of Art/Tampa, Museum of Art, 1996-1997), Cambridge (MA), 1996, p. 34-35.

18. Il Satiro Danzante, Roberto Petriaggi éd. (cat. expo., Rome, Camera dei deputati, Palazzo Montecitorio, 2003), Milan, 2003 ; Roberto Petriaggi éd., II Satiro Danzante Di Mazara del Vallo, il Restauro e I'Immagine, (colloque, Rome, 2003), Naples, 2005

19. Voir en particulier les synthèses très importantes de Caroline C. Mattusch, dont The Fire of Hephaistos, 1996, cité n. 17

20. Helmut Kyrieleis, Samos X. Der grosse Kuros von Samos, Mayence, 1996.

21. Niemeier, 2002, cité n. 6 .

22. Clemente Marconi, «Acrolithic and Pseudoacrolithic Sculpture in Archaic and Classical Greece and the Provenance of the Getty Kouros », dans Karol Wight éd., Cult Statue of a Goddess, (colloque, New York, 2007), Los Angeles, 2008, p. 4-13.

23. Brigitte Bourgeois, Philippe Jockey, « Polychrome Hellenistic sculpture in Delos. Research on surface treatments of ancient marble sculpture. Part I », dans Lorenzo Lazzarini éd., Interdisciplinary Studies on Ancient Stone - ASMOSIA VI, (colloque, Venise, 2000), Padoue, 2002, p. 497-506, et « [ ... ] Part II», dans Yannis Maniatis éd., ASMOSIA VII, (colloque, Thasos, 2003), à paraître.

24. Sur le rayonnement du Torse du Belvédère, malgré son état fragmentaire, voir le catalogue de I'exposition organisée à Munich, Der Torso: Ruhm und Rätsel, Raimund Wünsche éd., (cat. expo. Munich, Glyptothek/Rome, Musei Vaticani, 1998), Munich, 1998. Selon Giorgos Despinis, le torse représente probablement Philoctète : «Ancora una proposta di interpretazione per il torso del Belvedere ", dans Atti dell' Accademia nazionale dei Lincei. Rendiconti Classe di scienze morali storiche e filologiche $(R A L), 15 / 3,2004$, p. 393-408.

25. KlausFittschen, «ZurRekonstruktiongriechischer Dichterstatuen, 1 : Die Statue des Menander », dans $\operatorname{MDAl}(A), 106,1991$, p. 243-279.

26. Nick, 2002, cité n. 1.

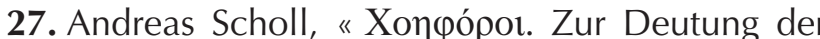
Korenhalle des Erechtheion », dans Jahrbuch des 
Deutschen Archäologischen Instituts [JDA/], 110, 1995, p. 178-212, et Die Korenhalle des Erechtheion auf der Akropolis: Frauen für den Staat, Francfort, 1998.

28. Giorgos Despinis, «Iphigeneia und Orestes. Vorschläge zur Interpretation zweier Skulpturenfunde aus Brauron", dans $\operatorname{MDAI}(A), 120,2005$, p. 241267.

29. Johannes Bergemann, Demos und Thanatos: Untersuchungen zum Wertsystem der Polis im Spiegel der attischen Grabreliefs des 4. Jahrhunderts v. Chr. und zur Funktion der gleichzeitigen Grabbauten, Munich, 1997.

30. Voir le site Internet du LIMC, accessible en français, anglais, allemand, italien, espagnol et grec : www.limcnet.org.

31. Le ThesCRA se compose actuellement de cinq volumes et d'un index publiés par le Getty Museum, Los Angeles, entre 2004 et 2006.

32. Joan Breton Connelly, Portrait of a Priestess: Women and Ritual in Ancient Greece, Princeton/ Oxford, 2007.

33. Voir, par exemple, Olga Palagia, William Coulson éd., Sculpture from Arcadia and Laconia, (colloque, Athènes, 1992), Oxford, 1993, et Regional Schools in Hellenistic Sculpture, (colloque, Athènes, 1996), Oxford, 1998.

34. Athens-Sparta, Nikolaos Kaltsas éd., (cat. expo., New York, Onassis Cultural Center, 2006-2007), New York, 2006.

35. Georgia Kokkorou-Alevras, «Laconian Stone Sculpture From the Eighth Century B.C. Until the Outbreak of the Peloponnesian War ", dans AthensSparta, 2006, cité n. 34, p. 89-94.

36. Johannes Bergemann, Demos und Thanatos: Untersuchungen zum Wertsystem der Polis im Spiegel der attischen Grabreliefs des 4. Jahrhunderts v. Chr. und zur Funktion der gleichzeitigen Grabbauten, Munich, 1997.

37. Andreas Scholl, «Av $\alpha \theta \eta \mu \alpha \tau \alpha \tau \omega v \alpha \rho \chi \alpha 1 \omega v$ : Die Akropolisvotive aus dem 8. bis frühen 6. Jahrhundert v. Chr. und die Staatswerdung Athens", dans Jahrbuch des Deutschen Archäologischen Instituts [JDAI], 121, 2006, p. 1-173.
38. Bernal, 1987 (1996), 1991, 2006, cité n. 2 ; Martin Bernal, D. Chioni Moore, Black Athena Writes Back: Martin Bernal Responds to His Critics, Londres, 2001 (en particulier le chapitre 12, p. 289307).

39. Jeremy Tanner, "Finding the Egyptian in Early Greek Art ", dans Roger Matthews, Cornelia Roemer éd., Ancient Perspectives on Egypt, (colloque, Londres, 2000), Londres, 2003, p. 132-139.

40. Voir, par exemple, Eric H. Cline, Diane HarrisCline éd., The Aegean and the Orient in the Second Millennium, (colloque, Cincinnati, 1997), (Aegaeumn 18), Liège/Austin, 1998.

41. Irene Bald Romano, «Early Greek Cult Images and Cult Practice ", dans Robin Hägg, Nanno Marinatos, Gullög C. Nordquist éd., Early Greek Cult Practices, (colloque, Athènes, 1986), (Svenska institutet, 4/38), 1988, p. 127-134.

42. Voir Cyprus and East Aegean: Intercultural Contacts from 3000 to 500 BC, (colloque, Samos, 2008), sous presse.

43. Mario Torelli, «L'ellenizzazione della società e della cultura etrusche", dans Gli Etruschi, Mario Torelli éd., (cat. expo., Venise, Palazzo Grassi, 20002001), p. 141-155.

44. Katja Lembke, Phönizische anthropoide Sarkophage, (Damaszener Forschungen, 10), Francfort, 2001.

45. Stucky, 1993, cité n. 3 ; Gabelmann, 1979 cité n. 3, p. 163-177; Robert Fleischer, Der Klagefrauensarkophag aus Sidon, Tübingen, 1983 ; Barbara Schmidt-Dounas, Der lykische Sarkophag aus Sidon, Tübingen, 1985 ; Marie-Theres LangerKarrenbrock, Der lykische Sarkophag aus der Königsnekropole von Sidon, Münster, 2000; Volkmar von Graeve, Der Alexandersarkophag und seine Werkstatt, Berlin, 1970; Wolfgang Messerschmidt, " Historische und ikonographische Untersuchungen zum Alexandersarkophag », dans Boreas, 12, 1989, p. 64-92 ; Renate Bol, «Alexander oder Abdalonymos? ", dans Antike Welt, 31, 2000, p. 585-599.

46. Voir, en particulier, Bonacasa, Buttitta, 1988, cité n. 4. 